J3eA, Journal sur l'enseignement des sciences et technologies de l'information et des systèmes,

Volume 3, Hors-Série 1, 22 (2004)

DOI : http://dx.doi.org/10.1051/bib-j3ea:2004622

(C) EDP Sciences, 2004

\title{
Aides techniques d'éducation gestuelle et conduite d'un fauteuil roulant électrique
}

J.P. Belheur ${ }^{1,2}$, A. Abellard ${ }^{3}$, G. Énéa ${ }^{3}$, P. Abélard ${ }^{3,4}$ et G. Nolibe ${ }^{5}$

${ }^{1}$ Institut de Rééducation Fonctionnelle Pomponiana Olbia, BP 41, F-83407 Hyères, France

${ }^{2}$ Association TROPHEE, piste des sambalettes, route de Valcros, F-83390 Cuers, France

${ }^{3}$ Laboratoire SIS/AI, Université de Toulon, BP 132, F-83957 La Garde CEDEX, France

${ }^{4}$ CERTV, IUT Toulon, BP 132, F-83957 La Garde CEDEX, France

${ }^{5}$ CESIGMA, Forum Aurélia, route du Val, F-83170 Brignoles, France 


\title{
Aides techniques d'éducation gestuelle et conduite d'un fauteuil roulant électrique
}

\author{
J.P. Belheur ${ }^{1,2}$, A. Abellard ${ }^{3}$, G. Enea ${ }^{3}$, P. Abellard ${ }^{3,4}$, G. Nolibe ${ }^{5}$ \\ ${ }^{1}$ Institut de Rééducation Fonctionnelle Pomponiana Olbia, BP 41, 83407 HYERES \\ ${ }^{2}$ Association TROPHEE, Piste des Sambalettes, Route de Valcros, 83390 CUERS \\ ${ }^{3}$ Laboratoire SIS/AI, Université de Toulon, BP 132, 83957 LA GARDE CEDEX \\ ${ }^{4}$ CERTV IUT Toulon, BP 132, 83957 LA GARDE CEDEX \\ ${ }^{5}$ CESIGMA, Forum Aurélia, Route du Val, 83170 BRIGNOLES
}

\begin{abstract}
Résumé - Pour amener l'enfant lourdement handicapé et souvent limité intellectuellement à apprendre comment utiliser fonctionnellement une manette pour la commande d'un fauteuil roulant électrique, l'ergothérapeute a défini une méthodologie d'éducation gestuelle. Elle repose sur l'utilisation de plusieurs aides techniques et permet une quantification des progressions / régressions. Pour valider les résultats, une plate-forme de tests appelée FRACAH a été réalisée. Elle comporte plusieurs systèmes de commande dont une manette gérée par un réseau de neurones qui compense tous les gestes incomplets et traite tous les gestes défectueux, dans un contexte de sécurité maximale. Cette solution a été retenue pour que le matériel s'adapte à l'enfant handicapé et non pas le contraire.
\end{abstract}

Mots clés : Auto-apprentissages rééducatifs, retour d'informations sensorielles, fauteuil roulant électrique, réseaux de neurones.

\section{INTRODUCTION}

Le fauteuil roulant électrique est un élément essentiel pour l'autonomie des personnes handicapées. Mais pour certaines, l'utilisation peut parfois être difficile, voire impossible, du fait de capacités physiques résiduelles trop faibles, d'une trop grande spasticité, d'une fatigabilité importante ou de troubles cognitifs. Par exemple, certaines personnes ne peuvent actionner qu'un simple capteur tout ou rien, ce qui rend difficile la commande d'un fauteuil roulant [1]. On peut alors apporter des améliorations à l'interface homme - machine standard de type joystick en utilisant des asservissements locaux ou des fonctionnalités intelligentes empruntées à la robotique: détection et évitement d'obstacles, accostage et passage de portes, suivi de parcours, planification de trajectoires... [2].

La robotique mobile de réadaptation et l'étude de fauteuils roulants intelligents s'apparentent au domaine de la robotique de service qui présente deux caractéristiques importantes: d'une part le robot partage son espace d'évolution avec les personnes et d'autre part, l'utilisateur n'est pas à priori compétent dans le domaine technologique, ce qui montre l'importance de l'interface homme - machine. La grande diversité des utilisateurs et des structures est une caractéristique importante de l'application. Par exemple, l'environnement peut être le domicile, un centre de réadaptation, un hôpital.... et l'encombrement potentiel des chemins ainsi que les distances à parcourir sont de ce fait, très variables. Par ailleurs, les patients en fauteuil ont des possibilités physiques et cognitives très différentes.

L'interface homme - machine doit donc être modulaire et configurable, tant du point de vue logiciel que des capteurs de commande. Elle doit être facile à mettre en place et apporter une sécurité maximale avec une grande fiabilité. Par ailleurs, pour optimiser le rapport coût / performances, il est important d'utiliser chaque fois que possible, du matériel non spécifique à l'application, comme par exemple, une base de fauteuil roulant du commerce.

Le projet FRACAH (Fauteuil Roulant A Commande Adaptée au Handicap) a été développé en respectant ces exigences.

\section{MATERIEL}

La base est un ancien fauteuil roulant 3 roues qui a été restauré mais n'importe quel fauteuil du commerce peut convenir. Elle fournit un support expérimental de tests de différentes solutions techniques d'aide au pilotage : bouton - poussoir, joystick, commande par la tête, par le pied, le souffle, la voix....(figure 1). Dans cet article, nous nous limitons à la présentation des différentes étapes chronologiques de rééducation gestuelle permettant à l'enfant IMC lourdement handicapé et souvent limité intellectuellement à apprendre comment utiliser fonctionnellement une manette pour la conduite d'un fauteuil roulant électrique.

\section{MÉTHODOLOGIE}

Pour éduquer régulièrement la main de l'enfant IMC, l'ergothérapeute doit impérativement procéder par étapes : étapes progressives, voire parfois régressives, dans lesquelles un certain nombre de paramètres doivent être pris en compte. Une méthodologie de quantification de l'évolution de l'apprentissage a donc été mise au point. Elle repose sur 5 phases. 


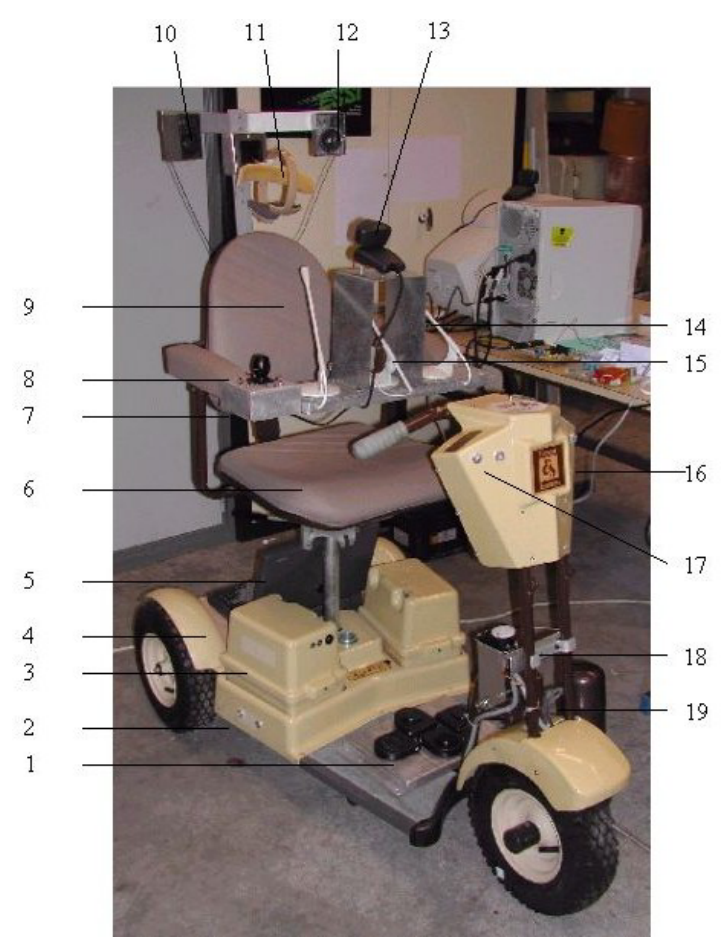

FRACAH : Fauteuil Roulant

A Commande Adaptée au Handicap

Plate-forme d'essais de solutions technologiques.

1. Commande par pédales

2. Détecteurs de proximité droite

3. Compartiment batteries

4. Compartiment cartes électroniques

5. Micro-ordinateur

6. Siège motorisé en rotation et élévation

7. Commande par manette neuronale

8. Voyants - guides directionnels

9. Commande par le souffle (Avant et Droite)

10. Haut-parleur droit (synthèse vocale)

11. Commande par la tête

12. Haut-parleur gauche (synthèse vocale)

13. Caméra (états des yeux, mouvements de tête, suivi du regard, expressions du visage), guidage automatique

14. Commande par le souffle (Arrière et Gauche)

15. Microphone pour commande vocale

16. Détecteurs de proximité Avant Gauche

17. Détecteurs de proximité Avant Droit

18. Servo-moteur de direction

19. Moteur de propulsion

Fig.1. Plate-forme FRACAH.

\section{A. Evaluation fonctionnelle des possibilités de la main de l'enfant}

Elle est réalisée simplement par un enregistrement graphique des zones gestuelles fonctionnelles (A) et des zones difficiles à atteindre (B) (figure 2).

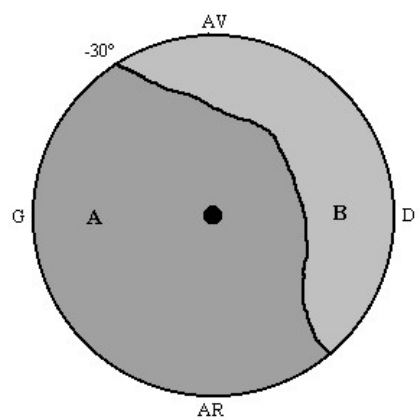

Fig.2. Evaluation fonctionnelle initiale avant rééducation.

\section{B. Chronologie rééducative pour la préparation au maniement de la manette}

C'est à partir de l'enregistrement précédent et de la parfaite connaissance que l'ergothérapeute a de l'enfant, qu'il va établir un projet personnalisé de travail progressif de la main par la mise au point d'une chronologie de rééducation conduisant au maniement optimal de la manette (figure 3).

\section{Exploitation de feedbacks multisensoriels}

Ils sont utilisés comme outils complémentaires de rééducation gestuelle pour motiver l'enfant dans la pratique des exercices définis par l'ergothérapeute. Tous les sens peuvent être sollicités : air pulsé sur le visage, vibreurs cutanés, animations sonores, parfums, distribution de bonbons, animations graphiques... Tous ces jeux sensoriels aident à l'orientation optimale du levier de la manette (figure 4).

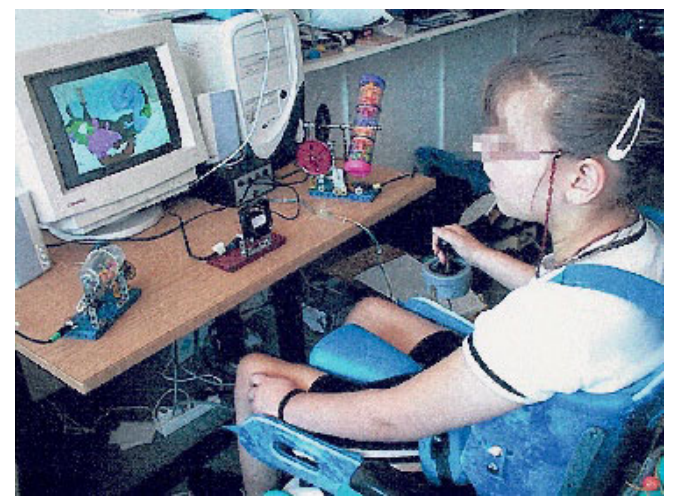

Fig.4. Jeux sensoriels. 


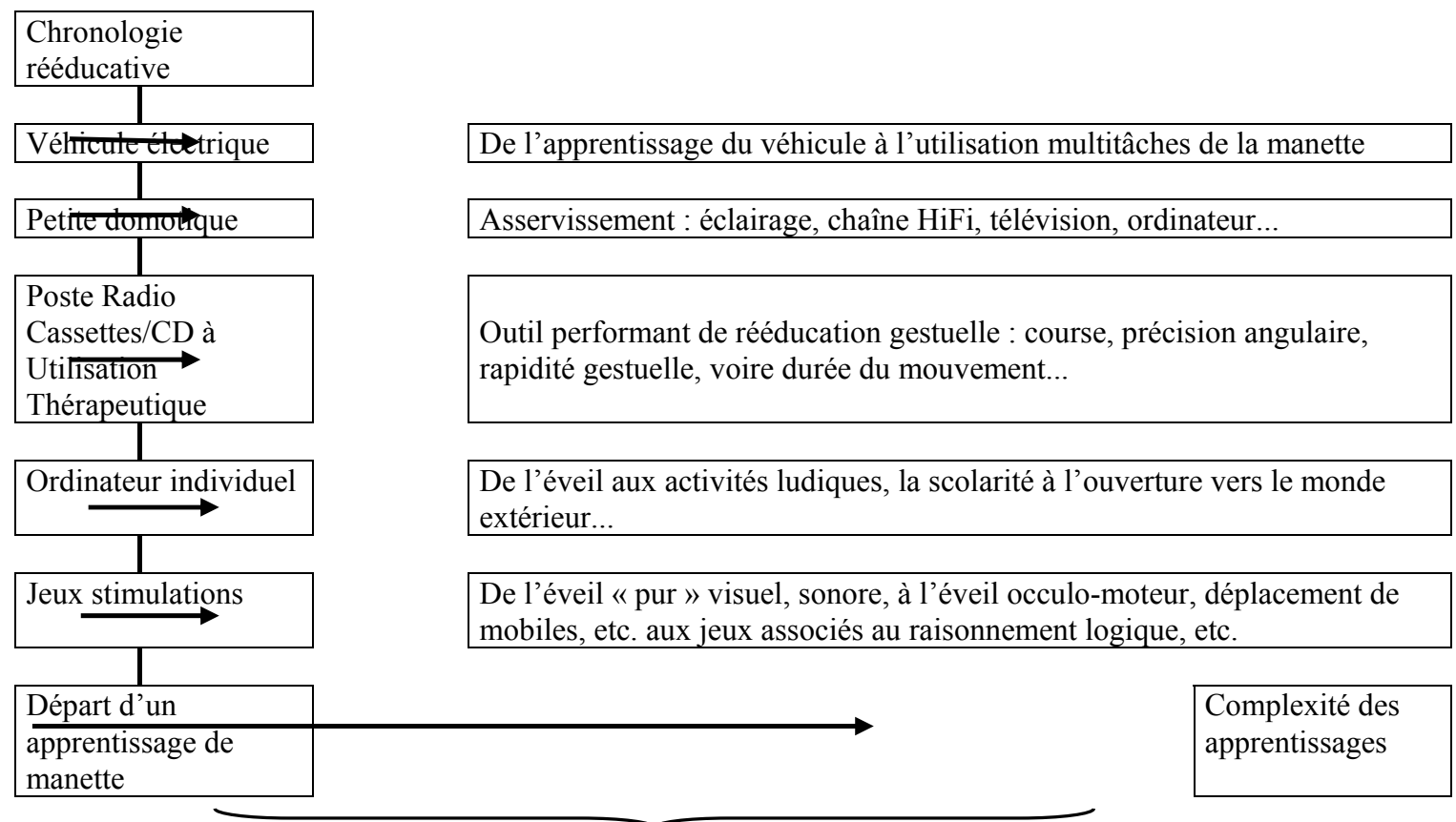

Ensemble personnalisé, adapté aux difficultés motrices, aux dyspraxies visuo-spatiales, et aux performances cognitives (adaptations techniques, dispositifs visuels, auditifs, vibratoires, etc.)

Fig.3. Etapes chronologiques de rééducation gestuelle.

D. Réglages progressifs des paramètres de fonctionnalités de la manette

La rééducation gestuelle est mise en place progressivement par adaptation des zones fonctionnelles à atteindre par chaque enfant (figure 5).

\section{E. Quantification et analyse des progrès / régressions}

Elle est réalisée régulièrement par enregistrement graphique des zones fonctionnelles (A), nonfonctionnelles (B) et d'évolution gestuelle (C) pour une comparaison avec les enregistrements antérieurs (figure $6)$.

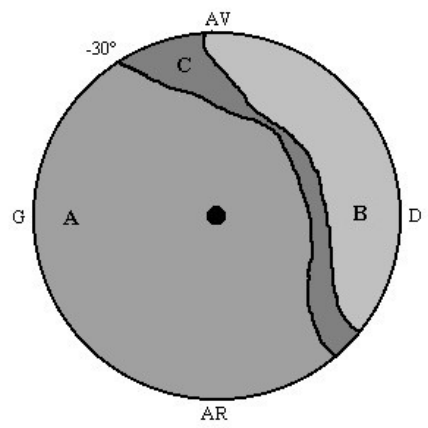

Fig.6. Analyse de résultats par superposition de zones.
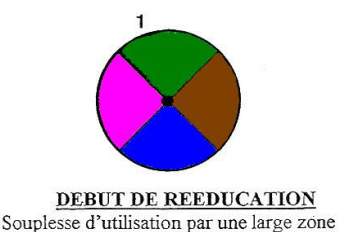
fonctionnelle.

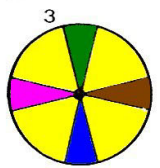

PROGRESSION REEDUCATIVE Poursuite de la rééducation par des valeurs angulaires de plus en plus restreintes.

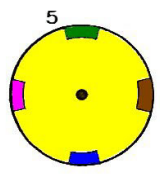

FIN DE REEDUCATION - la course du levier est à son maximum - le temps de latence peut être introduit.

- zone de REPOS du levier

zone programmée INACTIVE zone programmée ARRET

zone programmée ACTIVE zon programéc acrive \} (ici 4)

Fig. 5. Etapes progressives de rééducation gestuelle. 

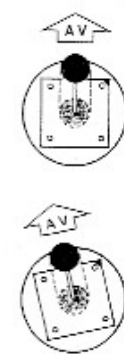

\section{(3)}

\section{$\uparrow$}

(2)

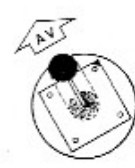

$\uparrow$

(1)

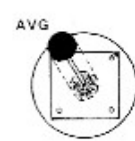

(0)

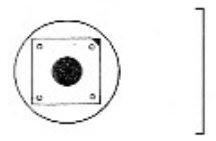

Fin de rééducation : La manette est dans l'axe du fauteuil. L'enfant a poursuivi ses progrès et a pu bénéficier d'un fauteuil personnel sans modification du positionnement de la manette, ce qui constitue un atout indéniable.

Apprentissage gestuel : Petit à petit, la manette est ramenée dans l'axe avant du fauteuil. L'enfant s'adapte aux changements successifs et imperceptibles sans dévier vers la gauche.

Avant rééducation : Afin de compenser le défaut d'utilisation de la manette, celle-ci a été orientée selon la prédominance gestuelle de l'enfant, permettant ainsi de diriger le fauteuil électrique vers l'avant.

Avant rééducation : La prédominance du geste pour utiliser la manette se situe vers la gauche $\left(-30^{\circ}\right)$, d'où l'impossibilité totale de se diriger vers l'avant.

Manette en position de repos. La boule de préhension reste centrée.

Fig.7. Exemple de stratégie d'apprentissage par rotation de la manette.

\section{APPLICATION AU PROJET FRACAH}

C'est l'ensemble de ces actions qui permet à l'enfant d'accéder à la commande manuelle du fauteuil électrique FRACAH (figure 7).

La manette est gérée par un réseau de neurones artificiels qui enregistre les limitations fonctionnelles de la main en phase d'apprentissage, puis qui compense tous les gestes incomplets et élimine les tremblements et les mouvements involontaires désordonnés.

En plus des capteurs actifs de sécurité, une caméra embarquée surveille l'état des yeux de l'enfant qui ne doit pas s'attarder trop longtemps à regarder sa manette, et les expressions de son visage (inquiétude, angoisse, peur...). Par ailleurs, un guidage visuel et sonore a été ajouté pour aider à la spatialisation de l'enfant. Un voyant est affecté à chaque direction sur la manette et une synthèse vocale fournit des ordres adaptés à chaque situation (figures 8 et 9).

\section{CONCLUSION}

La présence d'une centrale d'informations sensorielles facilite les apprentissages, aide à l'orientation optimale du levier de la manette, et fournit des aides à la compréhension. Cette approche accroît l'efficacité dans l'exécution des tâches à accomplir (rapidité, limitation des erreurs, diminution des répercussions liées au facteur émotionnel....) et améliore l'autonomie.
L'ensemble de ces actions doit permettre d'améliorer l'existant de façon significative car, pour certains jeunes IMC, ce n'est pas le temps qui aide le mieux à accéder à l'autonomie mais plutôt le choix des stratégies rééducatives mises en œuvre. L'électronique et l'informatique sont des outils qui nous conduisent à réfléchir et voir que d'autres personnes adultes lourdement handicapées, même avec des pathologies différentes peuvent elles aussi, accéder à l'autonomie. Les expériences pratiquées ont mis en évidence des difficultés liées à la dyspraxie visuo-spatiale lorsqu'elle est présente [3] et un travail de recherche est actuellement mené [4].

Pour les personnes présentant un handicap plus léger, l'approche innovante de ces travaux accroît l'efficacité dans l'exécution du geste (réduction du temps, limitation des erreurs, diminution du facteur émotionnel...) avec une sécurité maximale [5].

\section{BIBLIOGRAPHIE}

[1] G. BOURHIS, R. GELIN, A. PRUSKI : Robotique d'aide aux personnes handicapées. Applications non manufacturières de la robotique mobile. pp 193-238, Edition Hermès, 2000.

[2] M. BEN KHELIFA : Vision par ordinateur et robotique d'assistance. Application au projet MARH Mobile Autonome Robotisé pour Handicapés. Thèse de Doctorat, Université de Toulon, 2001. 
entrée informations prétraitées manette

entrées disponibles (en réserve)

entrées informations capteurs

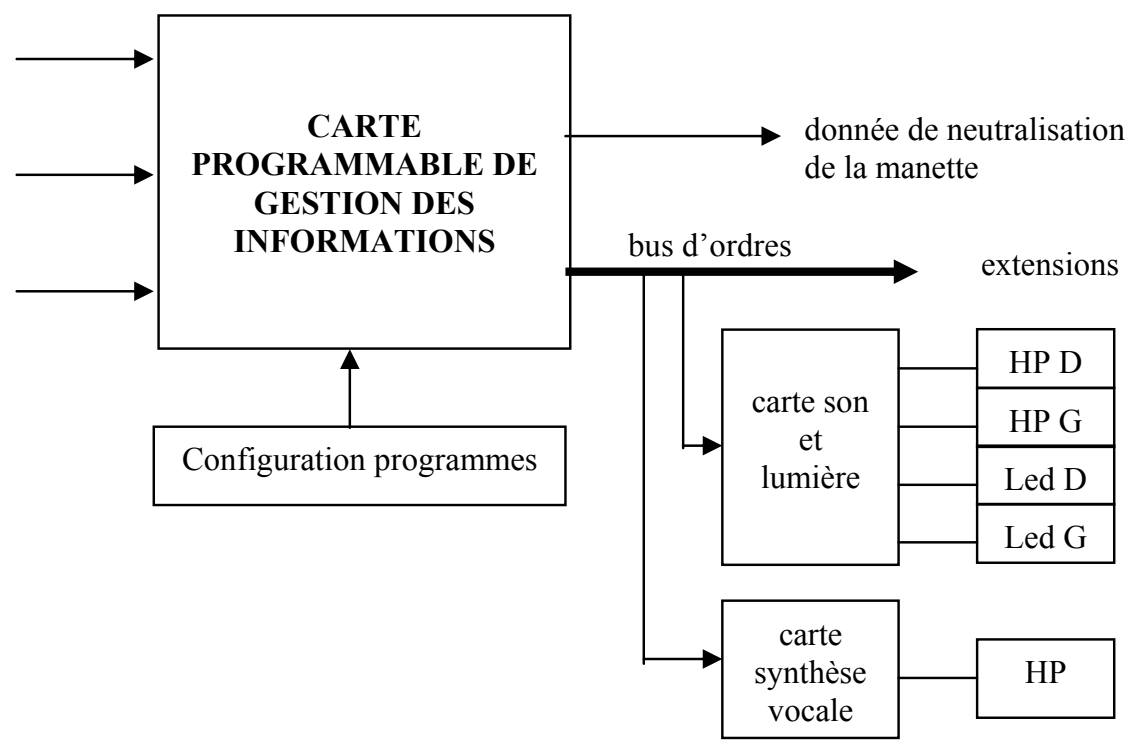

Fig. 8. Dispositif d'aides visuelles et acoustiques.

\begin{tabular}{|c|c|c|c|c|c|c|}
\hline Détecteur & AV & AR & AVD & AVG & $\mathrm{D}$ & $\mathrm{G}$ \\
\hline synchro. & & & & clignotement & & clignotement \\
\hline HP Droit & $\begin{array}{l}\text { son bi-ton } \\
\text { permanent }\end{array}$ & $\begin{array}{c}\text { Son } \\
\text { «crescendo » }\end{array}$ & & & & $\begin{array}{l}\text { son fréquence } \\
\mathrm{X} \text { fixe rythmé }\end{array}$ \\
\hline LED Gauche & & & clignotement & & clignotement & \\
\hline \begin{tabular}{|c} 
synchro. \\
HP Gauche
\end{tabular} & $\begin{array}{l}\text { son bi-ton } \\
\text { permanent }\end{array}$ & $\begin{array}{c}\text { Son } \\
\text { «crescendo » }\end{array}$ & & & $\begin{array}{l}\text { son fréquence } \\
\mathrm{X} \text { fixe rythmé }\end{array}$ & \\
\hline Synthèse vocale & recule ! & avance! & $\begin{array}{l}\text { recule et mets } \\
\text { la main du côté } \\
\text { de la lumière }\end{array}$ & $\begin{array}{l}\text { recule et mets } \\
\text { la main du côté } \\
\text { de la lumière }\end{array}$ & $\begin{array}{c}\text { va du côté du } \\
\text { son et de la } \\
\text { lumière }\end{array}$ & $\begin{array}{c}\text { va du côté du } \\
\text { son et de la } \\
\text { lumière }\end{array}$ \\
\hline
\end{tabular}

Fig. 9. Fonctions du dispositif d'aides visuelles et acoustiques.

[3] M. MAZEAU : Déficits visuo - spatiaux et dyspraxies de l'enfant. Collection Bois - Larris, Editions Masson, 1995.

[4] J.P. BELHEUR, M. PAILLOUX, B. PETITPREZ, A. ABELLARD, P. ABELLARD: Aides techniques d'éducation gestuelle pour enfant IMC. Handicap 2002, pp 185-186, 13-14 Juin 2002, Paris.

[5] A. ABELlARD, G. ENEA, P. ABELLARD, J.P. BELHEUR, G. NOLIBE: Aides techniques en appareillage pour handicapés : de l'éducation gestuelle au projet FRACAH. Colloque Recherche et Handicap, 5-6 Décembre 2002, Lyon.

\section{Remerciements}

Ces travaux sont soutenus financièrement par une bourse cofinancée Conseil Régional Provence Alpes Côte d'Azur et entreprise CESIGMA. Par ailleurs, nous remercions pour leur aide technique C. Reynaud, $\mathrm{T}$. Bonnelie, D. Peloux, S. Pignol et P. Arlotto de l'IUT de Toulon. 